\title{
Prevalence of extubation and associated risk factors at a tertiary care pediatric intensive care unit
}

\author{
Julia Simonassi, B.S. ${ }^{a}$ and Juan P. Bonora Sanso, B.S. ${ }^{a}$
}

\begin{abstract}
Introduction. Extubation failure (EF) is associated with a higher risk for pneumonia, hospital stay, and mortality.

Objective. To determine the prevalence of $\mathrm{EF}$ and identify the associated factors in our population. Materials and methods. Observational and retrospective design. All patients aged 1 month old to 18 years old who required mechanical ventilation for more than 12 hours and underwent at least one scheduled extubation during their stay in the 2012-2016 period were included. The aspects related to ventilation and the causes of failure were recorded.

Results. Seven hundred and thirty-one patients were extubated and the prevalence of EF was $19.3 \%$. The causes of failure included upper airway obstruction $(51.4 \%)$, muscle fatigue $(20.1 \%)$, respiratory center depression $(14.6 \%)$, and inability to protect the airways $(9.7 \%)$. As per the multivariate analysis, the independent factors that accounted for EF were neurological complex chronic condition (odds ratio $[\mathrm{OR}]=2.27$; $95 \%$ confidence interval $[\mathrm{CI}]=1.21-4.26$ ); acute lower respiratory tract infection in a patient with prior sequelae $(\mathrm{OR}=1.87,95 \% \mathrm{CI}=1.11$ 3.15); acute neurological injury ( $\mathrm{OR}=1.92,95 \%$ $\mathrm{CI}=1.03-3.57)$; unplanned extubation $(\mathrm{OR}=2.52$, $95 \% \mathrm{CI}=1.02-6.21)$, and presence of stridor $(\mathrm{OR}=5.84,95 \% \mathrm{CI}=3.66-9.31)$.

Conclusion. The main cause of EF was upper airway obstruction. Neurological sequelae, acute neurological injury, unplanned extubation, and the presence of postextubation stridor were identified as risk factors associated with EF.

Key words: tracheal extubation, mechanical ventilation disconnection, upper airway obstruction.
\end{abstract}

a. Hospital de Pediatría SAMIC

"Prof. Dr. Juan

P. Garrahan,"

Autonomous City of Buenos Aires,

Argentina.

E-mail address:

Julia Simonassi, B.S.: jsimonassi@garrahan. gov.ar

Funding:

None.

Conflict of interest: None.

Received: 3-15-2018 Accepted: 8-16-2018 of an artificial airway, sedation, and exposure to potential infections, make it imperative to reduce the length of use to prevent associated complications, including the resulting extended length of stay and increased costs. In addition, early measures may lead to extubation failure (EF), which is also associated with a higher risk for pneumonia, extended length of stay at the PICU, and mortality. ${ }^{1-3}$

The rate of EF is highly variable in the pediatric population and ranges from $2.7 \%$ to $22 \%$. Such diversity depends on the selected studied population, study design, and the different inclusion criteria. ${ }^{4}$ Several prognostic factors have been studied in the PICUs to prevent and reduce $\mathrm{EF}$, but results are not conclusive. . $^{5,6}$ The use of sedatives and analgesics, the length of assisted mechanical ventilation (AMV) use, patients' age, oxygenation disorders, hemodynamic instability, complex chronic conditions (CCCs), neurological injuries, nutritional or electrolyte balance disorders, and diaphragmatic dysfunction appear to increase the possibility of EF, either combined or individually.-9

Among the causes of EF, $40 \%$ are attributed to upper airway obstruction (UAO), which is the main reason of failure in the pediatric population. ${ }^{7}$ The causes of UAO may be dynamic, such as edema produced by endotracheal tube support and swallowing disorders, or structural, such as malformations, vocal cord paralysis, and granulomas. Predicting these before artificial airway removal is extremely difficult. An inadequate gas exchange, weak respiratory muscles, hemodynamic instability, and neurological injury are also causes of EF. ${ }^{4,10}$ 
It is important to know the risk factors associated with EF to implement measures aimed at reducing reintubation in patients with one or more EFs.

\section{OBJECTIVE}

To determine the prevalence of EF and identify failure-associated factors in our population.

\section{MATERIALS AND METHODS}

This was an observational, retrospective, analytical study conducted over 5 years, between January 1 1, 2012 and December 31 ${ }^{\text {st }}, 2016$ in a clinical-surgical PICU with 17 beds at a tertiary care hospital.

All patients aged 1 month to 18 years old who required orotracheal intubation connected to a mechanical ventilator for at least 12 hours and who were extubated at least once were included. Patients with a tracheostomy at the time of admission were excluded.

The physical therapists working at the PICU recorded data every day using standardized electronic cards; the first extubation was taken into consideration for those patients with more than one.

Outcome measures were recorded into four groups:

a. Demographic outcome measures: age (months old); sex; weight $(\mathrm{kg})$; Pediatric Index of Mortality 2 (PIM2); mortality; length of hospitalization in days; diagnosis at the time of admission, based on the following categories: 1. Trauma: multiple trauma, traumatic brain injury (TBI), gunshot wound, near-drowning, poisoning, electrocution. 2. Respiratory disorder in a previously healthy patient: acute lower respiratory tract infection (ALRTI), pneumonia, bronchiolitis. 3. Postoperative period of a general surgery: postoperative period of neck, chest, abdominal or limb surgery. 4. Neurosurgery: tumor resection, craniosynostosis, medullary decompression, hemispherectomy, drain placement, intracranial hematoma evacuation. 5. Immunocompromised patients: cancer, congenital or acquired immunodeficiency, bone marrow failure. 6 . Acute respiratory condition in a patient with prior sequelae: neurological and/or respiratory sequelae. 7. Non-respiratory infection: meningococcal disease, peritonitis, muscle abscess, cellulitis. 8. Acute neurological event: ischemic or hemorrhagic stroke, convulsive syndrome, encephalopathy, meningitis, hydrocephalus, arteriovenous malformation. 9. Neuromuscular conditions: botulism, myasthenia gravis, Barre-Guillain syndrome, muscular dystrophy, congenital myopathy, spinal atrophy. 10. Miscellaneous/other: obstructive sleep apnea syndrome, diabetic ketoacidosis, metabolopathy, mitochondrial disease, cardiovascular condition, dilated cardiomyopathy, asthma. CCC: a patient with an underlying respiratory, neurological, cardiac or oncological disease, airway or liver disorder, or any other disease that would condition his/her course during an acute process. Chronic or underlying disorder: a patient who required further clinical follow-up than a "healthy child." 11,12 Acute neurological injury: a patient with an altered level of consciousness and / or cognitive status that would affect the normal dynamics of airway protection mechanisms at the time of extubation (status epilepticus, delirium, abstinence). ${ }^{13-15}$

b. Airways: endotracheal tube (ETT) with or without balloon; ETT size; adequate ETT size as per Cole's formula (age in years $/ 4+4$ ); tracheostomy during hospital stay.

c. Ventilation and oxygenation disorders: days of $\mathrm{MV}$; acute respiratory distress syndrome(ARDS); prone position; use of high frequency oscillatory ventilation (HFOV); days of HFOV; days of muscle relaxants.

d. Weaning and extubation process: the decision to extubate was made by the treating team after performing a spontaneous breathing trial (SBT) with a T tube for at least 30 minutes. The SBT was considered positive if the patient did not develop any of the following conditions during the SBT: a. signs of respiratory distress, b. hypoxemia, c. hypercapnia, d. depression of consciousness (with ineffective breathing pattern), e. poor secretion management, f. other (e.g., seizures, hemodynamic alterations). If any of these situations occurred, the SBT was considered negative and the patient was not extubated and was reassessed the following day. EF: the need for endotracheal reintubation was considered in the first 72 hours after extubation. Unplanned extubation: it was defined as an extubation that had not been scheduled by the treating team (accidental extubation [AE] and selfextubation); the postextubation therapeutic approach was similar to the planned event. 
Causes of EF: UAO (involvement of the upper airways that resulted in two-phase respiratory distress, predominately inspiratory), poor airway protection (swallowing disorders and inability to mobilize secretions), muscle fatigue (increased respiratory rate and heart rate with signs of diaphoresis and intercostal retraction), hypoxemia, depression of consciousness (State Behavioral Scale $\leq-2) .{ }^{16}$ Stridor: it was classified in two categories, mild/moderate stridor (stridor without need for reintubation) and severe stridor (stridor that required reintubation).

Statistical analysis: the descriptive analysis was done using comparative tables and percentages; for continuous outcome measures, the median value was used as a measure of central tendency and the $25^{\text {th }}-75^{\text {th }}$ percentile, as a measure of dispersion. A non-parametric MannWhitney's test was used to compare numerical outcome measures. The $\chi^{2}$ test or Fisher's exact test were used for categorical outcome measures, as applicable. The odds ratio and the respective $95 \%$ confidence intervals were estimated. A multivariate logistic regression analysis was done with EF as the dependent outcome measure; independent outcome measures were selected if the $p$ value was $<0.1$ in the univariate analysis. The final model was developed using the maximum likelihood method and the stepwise elimination for outcome measures with a $p<0.1$ in each stage. The logistic regression model's calibration and discrimination were assessed using the Hosmer-Lemeshow test and the area under the curve (AUC) analysis. A $p$ value $<0.05$ was considered statistically significant for all tests. Data were analyzed using the IBM SPSS Windows software, version 20.0 (IBM Corp., Armonk, NY, USA).

\section{Ethical aspects}

The study was approved for publication by the Hospital Ethics Committee of Hospital de Pediatría "Prof. Dr. Juan P. Garrahan."

\section{RESULTS}

During the analysis period, 2280 patients were admitted to the PICU (Figure 1); of these, 1255 were excluded because they did not require AMV and 17, because they were older than

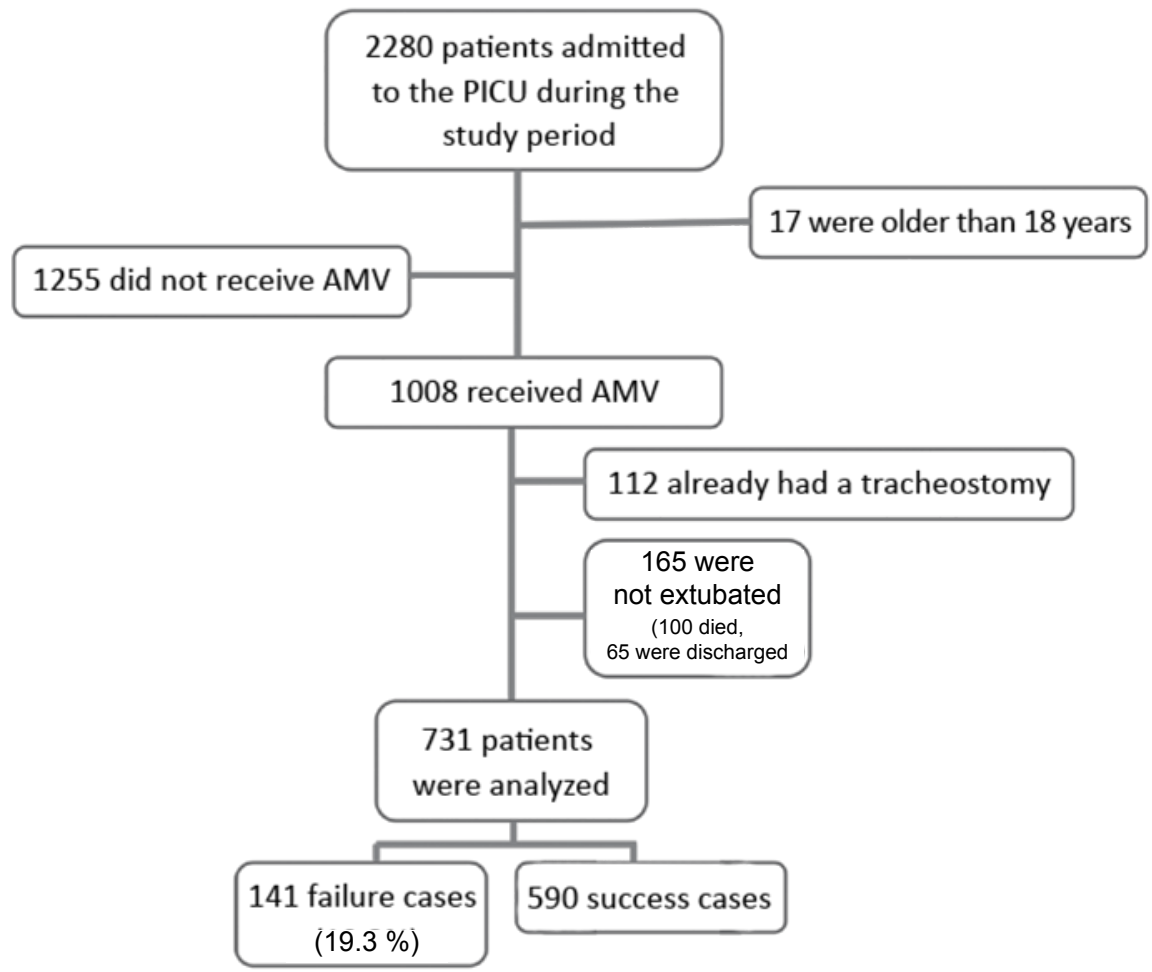

PICU: pediatric intensive care unit; AMV: assisted mechanical ventilation. 
18 years. Out of the 1008 patients who received AMV for at least 12 hours, 112 already had a tracheostomy tube and 165 were never extubated.

Therefore, the analysis included 731 patients and the prevalence of EF was estimated at $19.3 \%$ $(141 / 731)$.

No differences were observed in terms of weight, sex, and PIM2 score between extubation success and failure cases. Significant differences between these two groups were observed among patients with neurological CCC, ALRTI with prior sequelae, and acute neurological injury. Age did not show differences between patients with EF (median: 24 months old; interquartile

TABLE 1. Demographic outcome measures and univariate analysis of the risk factors for extubation

\begin{tabular}{|c|c|c|c|c|}
\hline No. of patients $(\%)$ & $\begin{aligned} & \text { Total } \\
\mathrm{n}= & 731(100)\end{aligned}$ & $\begin{array}{l}\text { Failure cases } \\
\mathrm{n}=141(19.3)\end{array}$ & $\begin{array}{l}\text { Success cases } \\
\mathrm{n}=590(\mathbf{8 0 . 8 )}\end{array}$ & p value \\
\hline Female sex, \% (n) & $45.8(335)$ & $46.1(65)$ & $45.7(270)$ & $\mathrm{n} / \mathrm{s}$ \\
\hline Weight in kg, Md[Q25-75] & $12[6.95,24.5]$ & $12[7,21.5]$ & $12[6.8,25]$ & $\mathrm{n} / \mathrm{s}$ \\
\hline Age in months, Md[Q25-75] & $24[6,96.5]$ & $21[7,85]$ & $24[6,104.5]$ & $\mathrm{n} / \mathrm{s}$ \\
\hline PIM2, Md[Q25-75] & $3.1[1.2,7.6]$ & $3.7[1.7,8.1]$ & $3[1.2,7.5]$ & $\mathrm{n} / \mathrm{s}$ \\
\hline \multicolumn{5}{|l|}{$\mathrm{CCC}, \%$ (n) } \\
\hline - Respiratory & $7.8(57)$ & $9.9(14)$ & $7.3(43)$ & $\mathrm{n} / \mathrm{s}$ \\
\hline - Neurological & $30.2(221)$ & $48.9(69)$ & $25.6(151)$ & $<0.05$ \\
\hline - Cancer & $5.3(39)$ & $2.8(4)$ & $5.9(35)$ & $\mathrm{n} / \mathrm{s}$ \\
\hline - Cardiac & $3.8(28)$ & $5(7)$ & $3.5(21)$ & $\mathrm{n} / \mathrm{s}$ \\
\hline - Other & $14(102)$ & $7.1(10)$ & $15.7(93)$ & 0.01 \\
\hline - No CCC & $38.9(284)$ & $26.2(37)$ & $42(247)$ & $\mathrm{n} / \mathrm{s}$ \\
\hline \multicolumn{5}{|l|}{ Diagnoses, \% (n) } \\
\hline - Trauma & $7(51)$ & $5(7)$ & $7.5(44)$ & $\mathrm{n} / \mathrm{s}$ \\
\hline - ALRTI in previously healthy child & $13.3(97)$ & $8.5(12)$ & $14.4(85)$ & $\mathrm{n} / \mathrm{s}$ \\
\hline - Post-operative period & $16.8(123)$ & $10.6(15)$ & $18.3(108)$ & 0.02 \\
\hline - Neurosurgery & $10.7(78)$ & $13.5(19)$ & $10(59)$ & $\mathrm{n} / \mathrm{s}$ \\
\hline - Immunocompromised patients & $2.3(17)$ & $1.4(2)$ & $2.5(15)$ & $\mathrm{n} / \mathrm{s}$ \\
\hline - ALRTI with prior sequelae & $23.4(171)$ & $34(48)$ & $20.8(123)$ & 0.001 \\
\hline - Non-respiratory infection & $7.7(56)$ & $5.7(8)$ & $8.1(48)$ & $\mathrm{n} / \mathrm{s}$ \\
\hline - Acute neurological event & $8.8(64)$ & $12.8(18)$ & $7.8(46)$ & 0.06 \\
\hline - Neuromuscular conditions & $3.1(23)$ & $4.3(6)$ & $2.9(17)$ & $\mathrm{n} / \mathrm{s}$ \\
\hline - Other & $7(51)$ & $4.3(6)$ & $7.6(45)$ & $\mathrm{n} / \mathrm{s}$ \\
\hline \multicolumn{5}{|l|}{ Univariate analysis } \\
\hline Acute neurological injury, \% (n) & $33.9(247)$ & $51.8(73)$ & $29.5(174)$ & $<0.001$ \\
\hline Unplanned extubation, \% (n) & $4.3(31)$ & $9.2(13)$ & $3.1(18)$ & 0.001 \\
\hline Acute respiratory distress syndrome, \% (n) & $14.4(105)$ & $23.1(31)$ & $12.6(74)$ & 0.004 \\
\hline Prone position, $\%(\mathrm{n})$ & $14.8(108)$ & $22(31)$ & $13.1(77)$ & 0.007 \\
\hline $\mathrm{HFOV}, \%(\mathrm{n})$ & $3.7(27)$ & $6.4(9)$ & $3.1(18)$ & 0.063 \\
\hline Stridor, \% (n) & $26(187)$ & $57.4(81)$ & $18(106)$ & $<0.001$ \\
\hline Tube with balloon, $\%$ (n) & $66.2(484)$ & $70(99)$ & $65.3(385)$ & $\mathrm{n} / \mathrm{s}$ \\
\hline Inadequate tube, $\%(\mathrm{n})$ & $23.8(174)$ & $19.9(28)$ & $24.7(146)$ & $\mathrm{n} / \mathrm{s}$ \\
\hline Days until extubation, Md[Q25-75] & $5[2,9]$ & $7[3,10]$ & $5[2,9]$ & 0.006 \\
\hline TV/weight (mL/kg), Md[Q25-75] & $9[7.4,11.4]$ & $9.21[7.3,11.4]$ & $9[7.5,11.5]$ & $\mathrm{n} / \mathrm{s}$ \\
\hline Days of muscle relaxants, Md[Q25-75] & $1[0,4]$ & $2[0,5]$ & $1[0,3]$ & 0.003 \\
\hline
\end{tabular}

$\mathrm{n}$ / s: not significant; Md: median; CCC: complex chronic condition; ALRTI: acute lower respiratory tract infection; HFOV: high frequency oscillatory ventilation; TV: tidal volume.

TABLE 2. Multivariate analysis of binary logistic regression

\begin{tabular}{lccc}
\hline Outcome measures & OR & 95 \% CI & P \\
\hline ALRTI with prior sequelae & 1.87 & $1.11-3.15$ & 0.018 \\
Neurological CCC & 2.27 & $1.21-4.26$ & 0.010 \\
Acute neurological injury & 1.92 & $1.03-3.57$ & 0.039 \\
Unplanned extubation & 2.52 & $1.02-6.21$ & 0.045 \\
Postextubation stridor & 5.84 & $3.66-9.31$ & $<0.001$ \\
\hline
\end{tabular}

OR: odds ratio; CI: confidence interval; ALRTI: acute lower respiratory tract infection; CCC: complex chronic condition. 
range [IQR]: 9-85) and those without EF (median: 24 months old; IQR: 6-108; $p=0.63$ ). A stratified analysis of this outcome measure compared the prevalence of EF in patients aged 6 months and younger $(\mathrm{n}=184 ; p=0.59), 7$ months- 1 year $(\mathrm{n}=98 ; p=0.76), 13$ months- 5 years $(\mathrm{n}=239$; $p=0.70)$, 5 years- 12 years $(\mathrm{n}=112 ; p=0.71)$, and 12 years and older $(\mathrm{n}=98 ; p=0.60)$. No significant differences were observed in any of the strata.

Table 1 shows the demographic outcome measures, the univariate analysis of risk factors, and a comparison of success and failure cases.

The reasons for EF were UAO in $51.4 \%$; muscle fatigue in $20.1 \%$; depression of consciousness in $14.6 \%$; inability to protect the airways in $9.7 \%$, and other causes, such as hemodynamic instability and hypoxemia, in $4.2 \%$.

There were 31 cases of unplanned extubation (4.3\%); of these, 7 remained extubated. The other 24 patients were reintubated and then 13 suffered a failed planned extubation. The reasons for failure were: 7 , upper airway obstruction; 2 , fatigue; 2 , inability to protect the airways; and 2 , impaired consciousness.

The univariate analysis showed that EF was associated with neurological CCC and other CCCs, ALRTI with prior sequelae, post-operative period of a general surgery; acute neurological injury; unplanned extubation; respiratory distress; HFOV requirement; prone position; stridor; longer use of AMV and muscle relaxants. These outcome measures were used in a multivariate logistic regression analysis (Table 2).

\section{DISCUSSION}

In our study, the prevalence of EF was established at $19.3 \%$. EF in pediatrics has been discussed by several authors but its prevalence has been described to vary between $2.7 \%$ and $22 \% .{ }^{17}$ It has been acknowledged that this may be due to different reasons, such as study design, studied population size, type and level of care at the intensive care unit, patient severity, medical history, comorbidities, and EF definition.

$\mathrm{EF}$ is described as the need of reintubation in a specific period, after extubation. Studies conducted by Thiagarajan et al., ${ }^{18}$ or Kurachek et al., ${ }^{4}$ defined failure with a period established at 24 hours; others like Baisch et al., ${ }^{19}$ at 48 hours; and finally, some like Edmunds et al., ${ }^{20}$ at the first 72 hours. In our ICU, we used the 72-hour period because it closely represented our daily practice.

Several authors ${ }^{5,13}$ observed in their studies that young age was a risk factor; therefore, an age-stratified analysis was done but no significant association with this outcome measure was noted in our population, like in the study by Baisch et al., ${ }^{19}$ where age was a risk factor only in the group of cardiac patients. It is also believed that this may be due to the high rate of patients with CCC in our ICU, compared to that in the study by Simon et al., ${ }^{12}$ whose population was older and at a high risk for EF.

Patients from different facilities of Argentina are referred to our ICU, so in this study we found a high percentage of patients with prior comorbidities, $61 \%$ in the general population, which increased to $74 \%$ among those with EF.

As observed, not all comorbidities appear to have the same effect on EF. Patients with neurological sequelae have a higher chance of EF than those with other types of chronic diseases. The inability to protect the airways and the higher possibility of having upper airway obstruction due to airway lability may be the main reasons for this. Salam et al. ${ }^{13}$ described that neurological patients, who have low peak expiratory flows or abundant secretions or inability to respond to simple orders (open the eyes, follow objects with the eyes, shake hands, and stick the tongue out), had a higher probability of EF, and the situation worsened if combined with the other three factors. Another significant association in this regard was the reason of admission to the ICU. The multivariate analysis indicated that a patient with CCC admitted due to a respiratory infection had a higher risk for EF. Simon et al., ${ }^{12}$ described that CCC considerably increased the number of admissions to the ICU, the length of stay in days, and the frequency of tracheostomy; for the latter, the frequency was up to 80 times higher than among patients without CCC. This situation gained importance considering that the pediatric population with CCCs has grown exponentially, probably due to survival in the neonatal period and the development of technology for chronic patient care.

Upper airway obstruction is the main cause of EF in the pediatric population. Newth et al., ${ }^{7}$ described it was the cause in $40 \%$ of cases. In our study, $51 \%$ of patients had EF due to upper airway obstruction. Several factors may have been related to these values, such as neurological disease, unplanned extubation, lack of sedation and analgesia protocols, and airway conditions. As described in the bibliography, no differences were observed in EF in relation to the use of a tube with or without balloon ${ }^{21-23}$ or larger-than- 
adequate tubes as per the formulas implemented in pediatrics. Baischet al., ${ }^{19}$ described pulmonary insufficiency, heart dysfunction, neurological disorder, muscle weakness, and excessive sedation as the causes of EF after UAO. In our study, muscle fatigue, impaired consciousness, and inability to protect the airways, respectively, were the reasons for EF.

Unplanned extubation resulted in an independent risk factor for EF in our study. In total, 31 cases were recorded, which accounted for $4.3 \%$ of patients with MV. Epstein et al., ${ }^{2}$ described a range of 3-16\%, while Boulain et al., ${ }^{24}$ found a prevalence of $10.8 \%$ in their study. In a pediatric study, Frank et al. ${ }^{25}$ described a range of 0.14-4.36 AEs/ 100 intubation days. On their side, Sadowskiet al., ${ }^{26}$ used the same index and found that their patients had 1.2 AEs/100 intubation days. In our population, we observed 0.56 AEs/ 100 intubation days.

A limitation of this study is that it was retrospective and conducted at a single site, so the studied population may have typical characteristics that prevent the extrapolation of results to other ICUs.

\section{CONCLUSION}

The prevalence of EF at a multipurpose PICU of a public tertiary care hospital was $19.3 \%$. The following independent risk factors for extubation were observed: ALRTI with prior neurological or respiratory sequelae, neurological CCC; acute neurological injury, unplanned extubation, and stridor after extubation.

\section{REFERENCES}

1. Cheifetz IM. Invasive and Noninvasive Pediatric Mechanical Ventilation. Respir Care. 2003; 48(4):442-53.

2. Epstein SK, Ciubotaru RL, Wong JB. Effect of Failed Extubation on the Outcome of Mechanical Ventilation. Chest. 1997; 112(1):186-92.

3. Epstein SK, Ciubotaru RL. Independent effects of etiology of failure and time to reintubationon outcome for patients failing extubation. Am J Respir Crit Care Med. 1998; 158(2):489-93.

4. Kurachek SC, Newth CJ, Quasney MW, Rice T, et al. Extubation failure in pediatric intensive care: A multiplecenter study of risk factors and outcomes. Crit Care Med. 2003; 31(11):2657-64.

5. Farias J, Alía I, Retta A, Olazarri F, et al. An evaluation of extubation failure predictors in mechanically ventilated infants and children. Intensive Care Med. 2002; 28(6):752-7.

6. Farias JA, Monteverde E. We need to predict extubation failure. J Pediatr (Rio J). 2006; 82(5):322-4.

7. Newth CJL, Venkataraman S, Willson DF, Meert KL, et al.
Weaning and extubation readiness in pediatric patients. Pediatr Crit Care Med. 2009; 10(1):1-11.

8. Fontela PS, Piva JP, Garcia PC, Bered PL, et al. Risk factors for extubation failure in mechanically ventilated pediatric patients. Pediatr Crit Care Med. 2005; 6(2):166-70.

9. Laham JL, Breheny PJ, Rush A. Do Clinical Parameters Predict First Planned Extubation Outcome in the Pediatric Intensive Care Unit? J Intensive Care Med. 2015; 30(2):89-96.

10. Khemani RG, Sekayan T, Hotz J, Flink RC, et al. Risk factors for pediatric extubation failure: Theimportance of respiratory muscle strength. Crit Care Med. 2017; 45(8):e798-805.

11. Feudtner C, Feinstein JA, Zhong W, Hall M, et al. Pediatric complex chronic conditions classification system version 2: Updated for ICD-10 and complex medical technology dependence and transplantation. BMC Pediatr. 2014; 14:199.

12. Simon TD, Berry J, Feudtner C, Stone BL, et al. Children With Complex Chronic Conditions in Inpatient Hospital Settings in the United States. Pediatrics. 2010;126(4):647-55.

13. Salam A, Tilluckdharry L, Amoateng-Adjepong Y, Manthous CA. Neurologic status, cough, secretions and extubation outcomes. Intensive Care Med. 2004; 30(7):1334-9.

14. Namen AM, Ely EW, Tatter SB, Case LD, et al. Predictors of successful extubation in neurosurgical patients. Am J Respir Crit Care Med. 2001; 163(3 Pt 1):658-64.

15. Cohn EC, Robertson TS, Scott SA, Finley AM, et al. Extubation Failure and Tracheostomy Placement in Children with Acute Neurocritical Illness. Neurocrit Care. 2018; 28(1):83-92.

16. Curley MA, Harris SK, Fraser KA, Johnson RA, et al. State Behavioral Scale: A sedation assessment instrument for infants and young children supported on mechanical ventilation. Pediatr Crit Care Med. 2006; 7(2):107-14.

17. Bankhead S, Chong K, Kamai S. Preventing extubation failures in a pediatric intensive care unit. Nurs Clin North Am. 2014; 49(3):321-8.

18. Thiagarajan RR, Bratton SL, Martin LD, Brogan TV, $\mathrm{t}$ al. Predictors of Successful Extubation in Children. Am J Respir Crit Care Med. 1999; 160(5 Pt 1):1562-6.

19. Baisch SD, Wheeler WB, Kurachek SC, Cornfield DN. Extubation failure in pediatric intensive care incidence and outcomes. Pediatr Crit Care Med. 2005; 6(3):312-8.

20. Edmunds S, Weiss I, Harrison R. Extubation failure in a large pediatric ICU population. Chest.2001;119(3):897-900.

21. Duracher C, Schmautz E, Martinon C, Faivre J, et al. Evaluation of cuffed tracheal tube size predicted using the Khine formula in children. PaediatrAnaesth. 2008; 18(2):113-8.

22. HuberBaur AM,Fuenmayor A, SalgarN, GottbergE.Factores de riesgo de extubación no planificada y reintubación en pacientes pediátricos. AVPP. 2013; 76(1):17-23.

23. Johnston C, de Carvalho WB, Piva J, Garcia PC, et al. Risk Factors for Extubation Failure in Infants With Severe Acute Bronchiolitis. Respir Care. 2010; 55(3):328-33.

24. Boulain T. Unplanned Extubations in the Adult Intensive Care Unit: a prospective multicenter study. Am J Respir Crit Care Med. 1998; 157(4 Pt 1):1131-7.

25. Frank B, Lewis R. Experience with intubated patients does not affect the accidental extubation rate in pediatric intensive care units and intensive care nurseries. Pediatr Pulmonol. 1997; 23(6):424-8.

26. Sadowski R, Dechert R, Bandy K, Juno J, et al. Continuous Quality Improvement: Reducing Unplanned Extubations in a Pediatric Intensive Care Unit. Pediatrics. 2004; 114(3):628-32. 